\title{
Linx
}

Revue des linguistes de l'université Paris X Nanterre

68-69 | 2013

Corpus et apprentissage du français

\section{Corpus et apprentissage du Français Langue d'Intégration (FLI)}

Hervé Adami et Virginie ANDRÉ

\section{(2) OpenEdition}

1 Journals

Édition électronique

URL : http://journals.openedition.org/linx/1535

DOI : 10.4000/linx.1535

ISSN : 2118-9692

Éditeur

Presses universitaires de Paris Nanterre

\section{Édition imprimée}

Date de publication : 19 novembre 2013

Pagination : 135-158

ISSN : 0246-8743

\section{Référence électronique}

Hervé Adami et Virginie ANDRÉ, "Corpus et apprentissage du Français Langue d'Intégration (FLI) »,

Linx [En ligne], 68-69 | 2013, mis en ligne le 19 novembre 2015, consulté le 10 décembre 2020. URL

http://journals.openedition.org/linx/1535 ; DOI : https://doi.org/10.4000/linx.1535 


\section{Corpus et apprentissage du Français Langue d'Intégration (FLI)}

Hervé Adami \& Virginie André, Université de Lorraine, ATILF

\section{Introduction}

Dans le domaine de la didactique du français, la structuration et linstitutionnalisation du champ de la formation linguistique des migrants, engagées dès le début des années 2000 (Adami, 2012a ; Leclercq, 2012), trouvent leur aboutissement dans le dispositif du Français Langue d'Intégration (FLI). Le FLI présente des spécificités notamment en termes d'enjeux et d'objectifs de formation ainsi qu'en termes de public. La raison essentielle de la mise en place de ce dispositif est l'amélioration de la qualité des prestations de formation assurées par les organismes de formation. L'enseignement du français est orienté par une démarche didactique particulière, nous le verrons, même si elle est proche de certaines méthodologies existantes.

Les outils et les ressources adaptés aux formations linguistiques des migrants manquent depuis la naissance de ce champ éducatif. Pour garantir la qualité de l'enseignement et de l'apprentissage du français aux migrants, l'utilisation de documents authentiques (cf. Holec, 1990) est toujours à l'ordre du jour. Néanmoins, il n'est pas toujours facile pour les formateurs de trouver sans cesse de nouveaux documents adaptés aux besoins spécifiques des apprenants. En outre, les corpus existants ne présentent que peu de données appropriées à ce type de formation. C'est notamment pour ces raisons que nous avons commencé à constituer un corpus, constitué de ressources écrites et multimédias recueillies en situation écologique, dans le cadre d'une recherche-action en collaboration avec un organisme de formation d'adultes migrants. 


\section{Le Français Langue d'Intégration}

\subsection{Le contexte socio-politique}

Le FLI apparaît dans un contexte de profonde transformation des vagues migratoires en France, tout comme en Europe. En effet, nous assistons désormais à une diversification des migrations, en ce qui concerne l'origine géographique des migrants mais aussi leur identité personnelle, sociale et professionnelle. Les projets migratoires et les flux sont multiples et s'inscrivent aujourd'hui dans un mouvement général de mobilité accrue (Wihtol de Wenden, 2008). Aujourd'hui, la majorité des migrants arrive en France dans le cadre du regroupement familial tandis que d'autres viennent dans l'espoir d'y trouver du travail ${ }^{1} ; d^{\prime}$ 'autres enfin fuient la crise ou la guerre dans leurs pays d'origine. Ces personnes arrivant sur le territoire français présentent également des caractéristiques socioculturelles diverses : nous rencontrons des individus peu scolarisés, très scolarisés, pas qualifiés, ou au contraire très diplômés, des travailleurs (légaux ou clandestins), des regroupements familiaux, des demandeurs d'asile politique, des victimes de la crise, etc.

Depuis les années 1980, il ne s'agit plus d'une immigration de travail constituée majoritairement d'hommes jeunes et seuls qui viennent travailler pour un temps limité puis repartent au pays : la «noria » des travailleurs que décrit Noiriel (1988) fait place à un mouvement d'implantation durable de familles qui s'installent et fondent leurs projets en France. La question politique et pratique de l'intégration se pose alors puisque les gouvernements successifs, mais également les immigrés eux-mêmes, ne pensent plus le retour au pays comme une issue inéluctable. Or, il apparait rapidement que la langue du pays d'accueil est un moyen incontournable pour favoriser cette intégration : la langue est perçue comme un instrument indispensable pour vivre au quotidien mais elle possède également une puissante charge symbolique d'acculturation.

La plupart des pays européens a mis en place des mesures politiques d'intégration des migrants (Extramiana, 2012), généralement avec une attention particulière pour la politique d'intégration linguistique. La maitrise de la langue devient une voie d'intégration, de cohésion sociale et une condition de réussite sociale et professionnelle. La politique d'intégration linguistique est liée, de façon plus générale, à la politique linguistique du pays d'accueil. En France, le monolinguisme officiel réduit les questions de choix de la langue d'intégration au français et, par la même occasion, simplifie les questions linguistiques, ce qui n'est pas le cas en Suisse ou à Québec, par exemple, où les questions d'intégration par la langue sont, de fait, plus complexes (Veillette, 2013). En outre, quel que soit le pays d'accueil, la maitrise de la langue intervient à différents niveaux dans le processus migratoire. Elle peut être une condition d'entrée sur le territoire, selon les pays, ou d'obtention d'une carte de séjour, de résident ou encore de la nationalité du pays d'accueil. De façon générale, l'apprentissage de la langue prend une place importante dans les débats sur la migration en Europe. C'est d'ailleurs une constante de la politique linguistique de la France dans le domaine de l'immigration. En 2001 déjà, sous le gouvernement Jospin,

${ }^{1}$ Immigrés et descendants d'immigrés en France, Edition 2012, INSEE. 
Christine Candide, une des responsables du FAS (Fonds d'Action Social) de l'époque, montrait l'importance de la maîtrise de la langue pour l'intégration des migrants et l'urgence d'une véritable politique de formation, professionnalisée et de qualité. Elle évoque un droit à la langue du pays d'accueil : «Il reste beaucoup de choses à faire, probablement à mieux caractériser ce droit et à interpeler au plus haut niveau. Nous sommes peut-être en train d'assister à l'émergence d'un nouveau droit de l'homme, à l'ère du post-modernisme. Aussi, si l'apprentissage de la langue est une obligation et donc un devoir, il constitue par là même la définition d'un droit » (Candide, 2001). On retrouvera une décennie plus tard l'auteure de ces lignes activement impliquée dans la mise en place du dispositif FLI. Mais avant cela, entre 2005 et 2007, cette orientation se concrétise avec la mise en place du Contrat d'Accueil et d'Intégration (CAI) et du Diplôme Initial de Langue Française (DILF) dont la fonction essentielle est de créer un dispositif de formation linguistique cohérent pour les migrants. En 2011, la mise en place du dispositif FLI vient parachever cette évolution et cette restructuration du champ de la formation linguistique des migrants dont l'histoire commence à s'écrire (Adami, 2009; Leclercq, 2012). Plusieurs alternances politiques gauche/droite sont survenues entre temps mais l'orientation en matière de politique de formation linguistique reste globalement la même ${ }^{2}$, démontrant en cela une continuité.

La formation linguistique des adultes migrants trouve ses origines dans les milieux militants (syndicats, associations) dès les années 1960. À partir des années 1980, ce champ éducatif se professionnalise et se structure peu à peu. La crise économique induit des besoins croissants en «formation de base » (Leclercq, 2007) pour les adultes faiblement ou non scolarisés qui peinent à retrouver un emploi. Concernant les migrants, c'est le CAI qui permet une structuration cohérente du domaine de la formation linguistique. Ce contrat engage les migrants à respecter les valeurs de la République et l'État à offrir aux migrants, dont le niveau de français est jugé insuffisant, une formation linguistique gratuite. Cette formation linguistique a pour objectif minimal l'obtention, par les migrants, du DILF. Le niveau de langue des primo-arrivants est évalué par un auditeur de l'Office Français de l'Immigration et de l'intégration (OFII). Si l'auditeur estime que la personne a besoin d'une formation linguistique, il l'oriente vers un organisme de formation, retenu dans le cadre du système des appels d'offre, qui prescrit aux migrants une formation linguistique de 400 heures maximum, selon l'objectif fixé : atteindre le niveau A1.1 ou passer de A1.1 à A.1 par exemple. La feuille de route du premier ministre (op. cit.) préconise désormais l'accès au niveau $\mathrm{A} 1$ au bout de la première année et au niveau $\mathrm{A} 2$ au bout de cinq ans.

L'introduction du FLI, piloté par la Direction de l'Accueil, de l'Intégration et de la Citoyenneté (DAIC) ${ }^{3}$, vient compléter le dispositif CAI/DILF, nous l'avons dit.

\footnotetext{
2 A l'heure où nous écrivons ces lignes, la feuille de route du premier ministre concernant la politique d'intégration réaffirme d'ailleurs très clairement la priorité donnée à la maitrise de la langue française, tant à l'école que pour les adultes. (Politique d'égalité républicaine et d'intégration. Fenille de route du gouvernement. Premier ministre, 11 février 2014.)

${ }^{3}$ La DAIC a changé de nom pour devenir, depuis octobre 2013, la Direction de l'accueil, de l'accompagnement des étrangers et de la nationalité (J.O. du 14/08/13). Elle sera toujours en charge de l'intégration linguistique des migrants.
} 
C'est d'abord et surtout un référentiel ${ }^{4}$ destiné aux organismes de formation professionnalisés et aux associations de bénévoles. Il constitue la base d'une démarche qualité, déjà mise en place d'ailleurs pour les centres de FLE. La spécificité de la formation des adultes migrants a conduit à l'élaboration d'un référentiel spécifique et donc de critères spécifiques d'évaluation et de certification des organismes de formation. Il encadre désormais les démarches pédagogiques et didactiques des formations linguistiques des adultes migrants.

\subsection{Le Français Langue d'Intégration: un nouveau dispositif pour un champ éducatif spécifique}

Comme nous l'avons expliqué précédemment, les nouvelles vagues migratoires en Europe entrainent une nouvelle réalité dans le champ de la formation linguistique des migrants. La France connaît également ces nouvelles configurations migratoires. Elle accueille aujourd'hui des migrants aux caractéristiques sociales, culturelles, économiques et professionnelles plus diversifiées qu'auparavant. Ainsi, si les migrants peu qualifiés, parfois analphabètes, arrivant en France dans l'espoir de trouver du travail puis de procéder à un regroupement familial sont encore nombreux, de nouveaux migrants, qualifiés, alphabétisés, parfois très diplômés, arrivent notamment des pays de l’Est de l'Europe dans l'espoir de trouver des conditions de vie meilleures que dans leur pays d'origine. Néanmoins, le faible niveau de scolarisation reste une spécificité importante des migrants. Plusieurs rapports nationaux ${ }^{5}$ le montrent et une étude internationale le confirme ${ }^{6}$ : "La population née à l'étranger dispose en moyenne d'un niveau d'éducation moins élevé que dans la moyenne des pays de l'OCDE, avec 24\% de diplômés du supérieur contre $31 \%$ dans les pays de l'OCDE ». Selon ces enquêtes, environ $60 \%$ des migrants présents en France possèdent un niveau inférieur ou égal au brevet des collèges français ou au niveau 5 de l'Éducation Nationale. Mais la comparaison est difficile entre les cursus scolaires européens et africains, par exemple, continent d'où proviennent de nombreux migrants en France. En effet, les scolarités effectuées en Afrique, par exemple, sont difficiles pour les élèves en raison des conditions matérielles, de la formation des enseignants, du matériel pédagogique mis à disposition et de l'organisation générale des institutions éducatives. Les niveaux affichés ou déclarés par les migrants, bien que déjà faibles, sont souvent en deçà du niveau supposé atteint. La faiblesse du niveau scolaire des migrants en France est une constante, même si ce niveau monte, comme le constatent les rapports cités plus haut. Or, cette caractéristique essentielle des apprenants migrants, la didactique du FLE/FLS ne sait pas la traiter. Bien davantage: elle ne l'a jamais intégrée à sa réflexion, ou bien de manière marginale ou ponctuelle.

\footnotetext{
${ }^{4}$ http://www.immigration.interieur.gouv.fr/Integration/L-apprentissage-du-francais/Le-label-qualiteFrancais-langue-d-integration/Le-referentiel-Francais-langue-d-integration-FLI.

${ }^{5}$ Immigrés et descendants d'immigrés en France, Edition 2012, INSEE, Fiche thématique « Education et maitrise de la langue »; Infos migrations, numéro 37, mars 2012, Département des statistiques, des études et de la documentation, Ministère de l'Intérieur, de l'Outre-Mer, des collectivités territoriales et de l'immigration; Beauchemin C. et al. (2010). Trajectoires et origine: enquête sur la diversité des populations en France, Premiers résultats. Document de travail 168. Teo, Insee et Ined.

${ }^{6}$ OCDE, Décembre 2012, Trouver ses marques : Les indicateurs de l'OCDE sur l'intégration des immigrés.
} 
Traditionnellement, la formation linguistique des migrants était associée, faute de mieux, au domaine de la didactique du Français Langue Etrangère (FLE) ou du Français Langue Seconde (FLS). Cependant, cette catégorisation ne convient pas aux adultes migrants, notamment parce qu'ils sont en immersion linguistique et qu'ils doivent s'approprier la langue dans un objectif d'intégration, nous y reviendrons. Nous ne détaillerons pas ici les raisons du caractère inapproprié des catégories telles que FLS ou encore Français sur Objectif Spécifique (FOS) puisque nous l'avons déjà fait par ailleurs (Adami \& André, 2012; Adami, 2012a) mais nous nous attarderons plutôt à montrer les spécificités du domaine de la formation linguistique des migrants adultes. C'est ce domaine professionnel et pédagogique, qui possède sa propre histoire, ses propres références, qui travaille avec des publics très différents des «étudiants» du FLE, dans un contexte économique et social concurrentiel où travaillent plus de cinq cents organismes de formation, c'est ce domaine donc que le référentiel et le dispositif FLI concernent. La spécificité n'est pas simplement didactique mais elle caractérise un champ professionnel radicalement différent. Néanmoins, si la formation linguistique des adultes migrants possède effectivement des caractéristiques très spécifiques, les bases théoriques et méthodologiques sont communes avec la didactique du FLE ou encore du FLS. Nous parlions déjà, il y a près d'une décennie, de «faux-jumeaux didactiques » (Adami, 2005).

Ce qui caractérise le dispositif FLI, et comme son appellation l'explicite sans ambiguité, c'est le fait qu'il inscrive la formation linguistique des migrants dans un parcours d'intégration économique, sociale et citoyenne. Or, cette notion d'intégration n'est pas consensuelle et suscite un débat politique, y compris parmi les didacticiens.

Au-delà du débat politico-scientifique autour de cette notion, il nous semble nécessaire de rappeler qu'il ne s'agit pas d'abord de se positionner "pour» ou «contre» l'intégration, ce qui n'a pas de sens à notre avis, mais de montrer que l'intégration des migrants est un processus social largement indépendant des débats que ce sujet provoque. Que l'on soit "pour» ou «contre » l'intégration, au nom, par exemple, de l'idéologie multiculturaliste, il n'en reste pas moins que les immigrés installés en France, et surtout leurs enfants et petits-enfants, s'intègrent et finalement s'assimilent à la société française. En effet, même si le principe de l'assimilation suscite encore davantage de levées de boucliers idéologiques, la réalité pourtant démontre que, générations après générations, les immigrés et leurs descendants se fondent dans l'ensemble social. C'est ce qu'a démontré, entres autres, l'enquête européenne EFNATIS (Schnapper, 2007). Que ce soit par les unions exogames, les pratiques linguistiques, vestimentaires, culinaires, de divertissement et même les pratiques religieuses qui tendent à se rapprocher de la moyenne de la population, l'intégration est en marche. Cette intégration n'est certes pas un long fleuve tranquille, si l'on pense, par exemple, aux discriminations ou, au contraire, aux fermetures identitaires sur la " culture » d'origine, mais elle s'effectue inéluctablement. Nous pensons à cet égard que les « freins » à l'intégration, pour reprendre un terme du débat public, ne sont pas d'ordre «culturel», si tant est que l'on puisse donner une définition sérieuse à cet adjectif, mais économiques et sociaux. En effet, dans le contexte de la crise 
permanente provoquée par la mondialisation libérale, toutes les populations, "autochtones » comprises, sont aux prises avec des difficultés telles que le modèle social ne peut plus résoudre. L'intégration est une notion, davantage qu'un concept, parce qu'une définition consensuelle et opératoire relève de la gageure. Nous concevons l'intégration, à la suite de Schnapper (2007), comme un «concept horizon». Cependant, si le «concept horizon » d'intégration est encore à travailler, il en va de même d'autres notions valises comme «culture » ou «identité», utilisés à satiété dans le domaine des sciences humaines, et tout particulièrement en sociolinguistique et en didactique des langues, sans qu'ils ne soient ni définis ni même interrogés de façon sérieuse. Or, le principe de l'intégration est souvent contesté au nom du respect des «cultures » d'origine et de «l'identité » culturelle des migrants. On mesure donc à quel point beaucoup de travail reste à accomplir dans l'élaboration de concepts opératoires.

Quant à la question particulière de l'intégration linguistique, qui nous intéresse en premier lieu ici, nous la concevons comme le processus sociolangagier d'acquisition de la langue dominante du pays d'accueil, en milieu social et/ou par le biais d'une formation, qui permet aux migrants allophones de comprendre et de se faire comprendre des locuteurs utilisant la langue dominante, c'est-à-dire d'interagir de façon efficace selon les situations dans lesquelles ils sont engagés et selon leurs objectifs de communication. L'intégration linguistique des migrants est ce processus qui élargit régulièrement le répertoire langagier des migrants et qui lui permet d'établir des contacts de plus en plus fréquents, denses et riches avec ses interlocuteurs et dans des situations de communication de plus en plus variées. L'acquisition de la langue dominante n'est pas un processus uniforme et rectiligne mais suit les tours et les détours des parcours socio-biographiques des migrants dans le pays d'accueil ${ }^{7}$. Par ailleurs, ce processus est très fortement influencé par les profils des migrants, selon leur niveau de scolarisation dans le pays d'origine surtout, mais également par leurs origines sociales, géographiques ou linguistiques. L'intégration linguistique étant un processus, il est impossible de déterminer un moment ou un niveau qui correspondrait à une intégration linguistique réussie. Tel migrant peut être parfaitement à l'aise dans les interactions en français avec ses collègues de travail mais éprouver des difficultés dès lors qu'il interagit avec les enseignants de ses enfants par exemple. Une intégration linguistique réussie se définit donc par rapport aux besoins des migrants et non dans l'absolu. Si un migrant peut comprendre et se faire comprendre de ses interlocuteurs dans toutes, ou presque toutes, les situations sociolangagières dans lesquelles il est engagé, on peut alors considérer que son intégration linguistique est réussie. Aucun locuteur, même natif et possédant un large répertoire langagier, n'est de toute façon en mesure de gérer sans problème toutes les situations langagières dans lesquelles il est engagé. Le processus d'intégration linguistique des migrants est donc en mouvement permanent.

\footnotetext{
${ }^{7}$ Nous menons actuellement un travail de recherche, qui a reçu le soutien de la DGLFLF, pour repérer et mesurer l'importance des différents facteurs qui contribuent à l'acquisition du français en milieu social (travail, réseau amical, voisinage, etc.)
} 


\subsection{Une démarche didactique spécifique}

Tout d'abord, il nous parait utile de rappeler que les apprenants concernés sont des adultes en situation d'immersion linguistique. Ainsi, la situation de formation en français ne représente qu'une partie du temps consacré à l'appropriation de la langue. En d'autres termes, la formation fait partie du processus d'apprentissage de la langue. Cette situation n'est pas sans conséquence sur la démarche didactique adoptée avec les migrants. Cette dernière se doit d'intégrer le fait que les migrants doivent pouvoir réinvestir au quotidien les acquis de la formation.

\subsubsection{Les objectifs des formations linguistiques des migrants adultes}

La particularité de ce public est qu'il apprend la langue dans le but de s'installer durablement dans le pays d'accueil et donc de pouvoir participer à toutes les situations de communication rencontrées habituellement par des natifs et de pouvoir effectuer les démarches nécessaires pour organiser sa vie en France. La formation linguistique vise donc bien l'autonomisation des migrants. Ces derniers doivent acquérir les compétences sociolangagières (Adami, 2010) qui leur permettront de s'intégrer à la société et d'y prendre pleinement part. En d'autres termes, les migrants doivent apprendre à participer à toutes les relations communicatives de la société : relations transactionnelles, interpersonnelles, professionnelles et médiatisées :

- les relations transactionnelles concernent toutes les interactions avec les services administratifs, les services sociaux, les relations de service ou encore les interactions dans les commerces ;

- les relations interpersonnelles sont les interactions avec les voisins, les amis ou encore les personnes rencontrées dans la rue ou à la sortie de l'école, par exemple ;

- les relations professionnelles sont les interactions que les migrants peuvent avoir sur leur lieu de travail ou lors de leur démarche de recherche d'emploi ou d'insertion professionnelle ;

- les relations médiatisées concernent ce que l'on peut appeler les interactions «tronquées », c'est-à-dire les médias, les affiches, les tracts, les panneaux mais aussi les boites vocales, les annonces dans les lieux publics ou encore les répondeurs téléphoniques.

Ainsi, les objectifs d'une formation linguistique à visée d'intégration, comme nous l'avons précisé auparavant, sont notamment de garantir trois aspects essentiels :

- l'intégration sociale permet aux migrants d'accéder à une autonomie socio-langagière. Ils apprennent à produire des discours adaptés à la situation de communication pour obtenir les effets pragmatiques escomptés. Ils peuvent alors gérer les aspects quotidiens de la vie en France : démarches administratives, achats, établissements de liens sociaux, gestion de la santé, scolarisation des enfants, connaissance du système éducatif, gestion des transports, etc. ; 
- l'intégration économique permet aux migrants de garantir leur insertion professionnelle ou leur sécurisation dans l'emploi ${ }^{8}$. Les migrants apprennent à découvrir et à se repérer dans le monde économique et dans le monde du travail (fonctionnement des entreprises, relations hiérarchiques, code du travail, droits et devoirs du salarié). Les organismes de formation linguistique sont d'ailleurs en contact permanent avec des structures d'insertion ou d'aide à l'emploi ;

- l'intégration citoyenne consiste à sensibiliser les migrants aux valeurs de la société d'accueil, en l'occurrence pour la France, aux principes de la République (liberté, égalité, fraternité, démocratie, laïcité, droit à l'instruction, à l'expression, au vote, au respect des salariés et du droit du travail, de la vie privée, du corps humain, etc.) et plus largement aux principes du « vivre ensemble ».

Le public migrant en formation linguistique est extrêmement hétérogène mais il a la particularité d'être en insécurité langagière dans le pays dans lequel il a choisi de vivre. En effet, il lui est souvent difficile d'effectuer les actions les plus simples, telles que lire un panneau de signalisation, une facture, prendre un rendez-vous chez un médecin, comprendre les horaires d'ouverture de la mairie ou demander son chemin. Un des objectifs des formations linguistiques à visée d'intégration est de réduire cette insécurité langagière et de donner la possibilité aux migrants d'évoluer de plus en plus aisément dans la société.

\subsubsection{La méthodologie didactique en Français Langue d'intégration}

La méthodologie du FLI est en partie liée à la méthodologie du FLE telle qu'elle peut être définie, notamment, par Cuq et Gruca (2002). Les grands principes de l'approche communicative, de la perspective actionnelle, de l'approche par compétences, de l'individualisation, de la modularisation ou encore de la pédagogie par objectifs de la didactique des langues sont réinvestis et ajustés dans le cadre de la formation linguistique pour les adultes migrants. Dans le cadre du principe de la contextualisation de l'enseignement/apprentissage des langues, nous nous attarderons ici sur l'utilisation des documents authentiques pendant les formations. Le champ de la formation linguistique des migrants a depuis longtemps adopté l'utilisation de documents authentiques pour les raisons déjà évoquées plus haut et résumées ici : les migrants sont en immersion linguistique dans le pays d'accueil et doivent comprendre les discours présents dans cette société afin de pouvoir y vivre (Adami, 2009).

Il s'agit alors d'envisager de nouvelles façons d'apprendre en lien avec le contexte, nécessairement pris en compte dans la formation d'un public en immersion. Nous pouvons préciser également que les documents authentiques peuvent faire leur apparition en formation à l'initiative des apprenants. Il est fréquent qu'une personne vienne en séance de formation avec un dossier à remplir, une facture à comprendre, un formulaire à compléter... Les documents authentiques représentent non seulement un moyen d'apprentissage du français mais également une fin, un objectif à part entière.

${ }^{8}$ Le FLI peut être articulé ici avec le Français Langue Professionnel (FLP), voir Mourlhon-Dallies (2008). Le Centre Européen des Langues Vivantes du Conseil de l'Europe mène également un projet qui vise à «développer les compétences langagières des migrants sur le lieu de et pour le travail» (http://www.ecml.at/I2/tabid/891/language/fr-FR/Default.aspx). 
Pour assurer les formations linguistiques, chaque formateur doit donc se constituer une banque de données de documents authentiques exploitables en situation de formation, c'est-à-dire destinés à servir de supports à des activités pédagogiques, et appropriée aux objectifs de la formation. Cependant, nous le rappelons, une des spécificités des formations en FLI est la grande hétérogénéité du public et, par conséquent, des objectifs de formation ou des activités à proposer. Les causes de cette hétérogénéité sont de différentes natures, dont les principales ont été listées dans Adami (2012b) : les origines géographiques, les origines sociolinguistiques, le niveau de scolarisation dans le pays d'origine, les origines sociales, le parcours dans le pays d'accueil, les parcours individuels de vie. Adami (Ibid. : 77) explique également que les formateurs ont tendance à appliquer «la règle empirique du plus petit dénominateur commun en termes d'objectif, de progression ou d'activités » et ajoute que «cette règle non dite qui vise à satisfaire les besoins du plus grand nombre d'apprenants, ne satisfait en réalité personne : les uns peinent pour ne pas décrocher, tandis que les autres s'ennuient» (Ibid.). Cette pratique va à l'encontre de la nécessité de prendre en compte les besoins tout à fait spécifiques de chaque individu. La méthodologie didactique mise en œuvre grâce à la création d'un corpus de documents authentiques permet de proposer aux formateurs un panel de documents et d'activités par niveau (ceux du Cadre européen commun de référence pour les langues - CECRL) pouvant être choisis en fonction de chaque apprenant, nous le préciserons dans les points suivants. En outre, les contraintes matérielles qui pèsent sur les organismes de formation et sur les formateurs expliquent également la nécessité d'un corpus de documents sociaux, dont les supports sont accessibles, décrits, classés et échantillonnés.

\section{La constitution du corpus}

\subsection{Le cadre d'une démarche de recherche-action}

Notre intervention s'inscrit dans le cadre d'une recherche-action. Nous sommes engagés sur le terrain de la formation linguistique des adultes migrants depuis de nombreuses années et nous entretenons des contacts permanents avec les apprenants, les formateurs, les coordinateurs pédagogiques, les équipes de direction et tous leurs partenaires (OPCA, services de l'État chargés de la mise en place des formations linguistiques, structures relais de la formation, etc.). Nous avons explicité les principes de notre démarche (Adami \& André, 2012) selon laquelle nous concevons notre travail de recherche comme une implication distanciée sur le terrain. Nous pensons que quelques incursions ou quelques « descentes » sporadiques sur le terrain non seulement ne suffisent pas, mais qu'elles sont même contre productives parce qu'elles ne donnent à voir au chercheur qu'une fraction, et surtout qu'un moment, de la réalité du domaine de la formation d'adultes. Notre implication concernant la mise en place du corpus de documents sociaux pour la formation linguistique des migrants, que nous présentons ici, s'inscrit donc dans un long travail de proximité avec le terrain et ses acteurs, qui a donné lieu aux publications auxquelles nous avons déjà renvoyé le lecteur au long de cet article. La réalisation de l'outil dont il est question ici est une co-construction entre 
notre groupe de recherche et l'organisme de formation Formabilis qui porte le projet. Ce travail de mise en place nous a d'ores et déjà permis de recueillir des données concernant la façon dont les formateurs ont perçu le projet, sur la façon dont ils se sont engagés et sur leur vision de la réalisation du projet. Ces données donneront lieu à des publications ou à des communications dans un autre cadre que celui-ci puisque nous nous concentrons ici sur la question du corpus proprement dit. En revanche, ce travail de recherche-action a pour objectif de tester la mise en pratique de cet outil et de recueillir toutes les données nécessaires pour notre recherche: perception des apprenants et des formateurs, obstacles techniques, problèmes didactiques ; adéquation ou limite des documents; etc. Cette phase de notre travail de recherche-action n'a pas débuté puisque l'outil est encore en cours de réalisation et de finalisation. Notre contribution dans cet article concerne donc la phase centrale de la recherche-action, c'est-à-dire la réalisation de l'outil et la constitution du corpus.

Cette méthode de recherche possède, selon nous, deux avantages déterminants : d'une part, elle nous permet de tester en temps réel et en direct des innovations pédagogiques, des outils, des démarches qui, sans cela, ne resteraient que d'abstraites propositions théoriques sans portée opérationnelle et ne dépasseraient pas le cercle du débat entre chercheurs; d'autre part, les résultats de ces recherches sont directement transférables sur le terrain et peuvent permettre aux organismes de formation et aux acteurs, non seulement de bénéficier des recherches, mais d'y participer en devenant eux-mêmes des praticiens chercheurs, voire des chercheurs tout court, si l'on pense à une formatrice qui a décidé de faire une thèse de doctorat qu'elle doit prochainement soutenir sous notre direction.

\subsection{Un corpus de documents sociaux pour la formation linguistique à visée d'intégration}

De multiples initiatives sont prises aujourd'hui pour collecter, informatiser, exploiter et partager des données en langues. Dans ce cadre, un corpus de français de référence est lentement en train de se constituer ${ }^{9}$ mais il laisse de côté des pans entiers de la réalité des pratiques sociolangagières. En effet, si le corpus des interactions interpersonnelles est assez important ${ }^{10}$, il n'existe en revanche que peu de données significatives d'interactions transactionnelles et professionnelles, encore moins de données de productions orales automatisées ou standardisées (boîtes vocales ou annonces publiques par exemple) et d'écrits urbains et professionnels. Or, c'est cette réalité sociolinguistique que vivent les migrants en insécurité langagière. C’est ce français d'intégration que nous avons commencé à recueillir pour en constituer un corpus de données écrites et multimédias (audio et vidéo).

\footnotetext{
${ }^{9}$ Nous faisons référence ici au projet ORFÉO (Outils et Ressources pour le Français Ecrit et Oral), retenu dans le cadre de la programmation «Corpus et outils de la recherche en SHS » de l'Agence Nationale de la Recherche (ANR). Si ce corpus n'est pas conçu en vue d'une exploitation pédagogique, il parait tout à fait possible de pouvoir l'utiliser à cette fín.

10 Certains formateurs linguistiques ont déjà puisé, par exemple, dans le corpus TCOF (Traitement de Corpus Oraux en Français) constitué à Nancy (André \& Canut, 2010) des extraits de conversations quotidiennes ou de consultations médicales pour les utiliser avec des publics migrants et/ou peu qualifiés.
} 


\subsection{L'outil FLIREP}

L'objectif premier de ce corpus est de combler un manque d'outils et de ressources dont pourraient disposer facilement les formateurs FLI. Il existe bien des matériels pédagogiques (des manuels, par exemple), ceux édités entre 1953 et 2009 ont d'ailleurs été recensés par l'association VA Savoirs dans le cadre du projet MALIN (Mutualisation et Analyse des ressources pour la formation LINguistique des adultes peu ou pas francophones et/ou peu ou pas scolarisés) ${ }^{11}$. Néanmoins, peu sont adaptés à une démarche FLI. L'utilité d'une plateforme collaborative de données authentiques pour la formation linguistique des migrants a déjà été soulevée (Gloaguen-Vernet, 2009) mais un tel outil n'avait pas encore vu le jour.

La conception scientifique et la réalisation matérielle de ce corpus ont été faites en collaboration entre le groupe de recherche Langage, Travail et Formation ${ }^{12}$ du laboratoire ATILF (Université de Lorraine \& CNRS) et l'organisme de formation Formabilis ${ }^{13}$, qui porte le projet, et avec le soutien de la DAIC. Ce corpus est intitulé FLIREP : Français Langue d'Intégration : Ressources et Exploitations Pédagogiques et prend la forme d'un site Internet ${ }^{14}$. Le site est composé de deux parties : des ressources et des exploitations pédagogiques associées aux ressources. Un corpus « d'amorçage » a été constitué pour permettre l'utilisation rapide de FLIREP dès sa création ${ }^{15}$ mais ce site vise la mutualisation, entre formateurs, de ressources et d'activités pédagogiques.

Au-delà de la constitution d'un corpus, les données concernant les contextes sociaux de production et de réception du français permettent d'atteindre deux objectifs principaux. Le premier est l'analyse (socio)linguistique (textuelle, interactionnelle ou encore pragmatique) de ces discours et échanges langagiers particuliers. Cette analyse donne accès à une description plus fine de leurs caractéristiques, encore peu étudiées. Le second objectif, intimement lié au premier, vise l'exploitation de ces données, ainsi que leur analyse, dans des contextes d'apprentissage du français. Les données peuvent être analysées en vue de saisir les compétences langagières des locuteurs en situation et peuvent ainsi être utilisées à des fins pédagogiques.

\section{4. Échantillonnage du corpus et métadonnées}

La conception de la plateforme de données a permis de réfléchir plus précisément à l'échantillonnage du corpus afin de viser le recueil d'une variété de documents appropriés. Tous les documents circulant dans la société française sont potentiellement exploitables pour la formation linguistique des migrants. Néanmoins, ces documents doivent être catégorisés et classés pour faciliter leur interrogation automatique et leur sélection par les formateurs.

${ }^{11}$ L'ensemble des travaux de ce projet est présenté à l'adresse suivante : http://malin.over-blog.org/.

12 En plus de deux co-auteurs de cet article, Florence Poncet a participé à la création ce corpus.

13 http://www.formabilis.fr/. Nous tenons à ce propos à remercier ici Formabilis, sa direction et ses salariés, très impliqués dans les projets de recherche et d'innovation pédagogique que nous menons.

14 http:/ / flirep.formabilis.fr/

${ }^{15}$ Le corpus d'amorçage a également été constitué afin de servir d'exemple, pour les formateurs, en termes de types de documents, de qualité des documents mutualisables ainsi qu'en termes de types d'exploitations pédagogiques. Ce corpus d'amorçage participe à la recherche d'homogénéisation des formations mentionnée plus haut. 


\begin{tabular}{|c|c|}
\hline Thèmes & Sous-thèmes \\
\hline \multirow[t]{9}{*}{ Consommation et services } & Abonnements \\
\hline & Achats courants \\
\hline & Après-vente \\
\hline & Impôts \\
\hline & Paiements \\
\hline & Restauration \\
\hline & Services bancaires \\
\hline & Services postaux \\
\hline & Travaux \\
\hline \multirow[t]{6}{*}{ Emploi, vie professionnelle } & Chercher un emploi \\
\hline & Courrier \\
\hline & Démarches administratives \\
\hline & Fiches de paie \\
\hline & Sécurité \\
\hline & Vie professionnelle \\
\hline \multirow[t]{4}{*}{ Logement } & Cohabiter \\
\hline & Déménager \\
\hline & Etre un éco-citoyen \\
\hline & Gérer son logement \\
\hline \multirow{5}{*}{ Loisirs } & Cuisine \\
\hline & Culturel \\
\hline & Excursions \\
\hline & Loisirs sportifs \\
\hline & Sorties \\
\hline \multirow[t]{5}{*}{ Premiers échanges } & Faire connaissance \\
\hline & Parler de sa famille \\
\hline & Parler de soi \\
\hline & Parler des autres \\
\hline & Se présenter \\
\hline \multirow{2}{*}{ S’orienter, se déplacer } & S'orienter dans son environnement \\
\hline & Se déplacer \\
\hline \multirow[t]{4}{*}{ Santé } & Consultations \\
\hline & Hôpital \\
\hline & Pharmacie et médicaments \\
\hline & Sécurité sociale et mutuelles \\
\hline \multirow[t]{3}{*}{ Scolarité } & Correspondance \\
\hline & Inscriptions \\
\hline & Vie scolaire \\
\hline \multirow[t]{4}{*}{ Situer dans le temps } & Dates et horaires \\
\hline & Durées, rythmes et fréquences \\
\hline & Météo \\
\hline & Rendez-vous \\
\hline \multirow[t]{2}{*}{ Transports } & Transports privés \\
\hline & Transports publics \\
\hline \multirow[t]{3}{*}{ Citoyenneté et vie en France } & Administration \\
\hline & Vie quotidienne \\
\hline & Institutions \\
\hline
\end{tabular}

Tableau 1 : Liste des thèmes et sous thèmes du corpus FLI 
Un premier échantillonnage thématique a donc été réalisé en fonction des objectifs des formations linguistiques. Le Tableau 1 présente les thèmes et sous-thèmes retenus et qui serviront de métadonnées interrogeables par l'intermédiaire de l'interface d'interrogation de FLIREP. Une première expérimentation de FLIREP, par le biais des formateurs qui «testent» les outils en situation d'apprentissage réelle avec leurs apprenants, montre que certains thèmes et sous-thèmes ne sont pas toujours pertinents (s'orienter dans son environnement vs. se déplacer, par exemple) et que certains documents entrent dans plusieurs catégories. Une légère modification des métadonnées est en cours de réalisation, au vu des résultats de cette expérimentation.

Ensuite, les documents sont catégorisés selon leur nature : écrit, audio, vidéo et visuel. Si les formations FLI visent un niveau supérieur à l'oral par rapport à l'écrit ${ }^{16}$, cela ne signifie pas que le corpus doive comporter plus de documents oraux qu'écrits. Au contraire, le travail des compétences à l'écrit, prenant souvent plus de temps en formation que celui de l'oral, doit pouvoir se faire à partir d'un nombre important de documents variés. Ce travail est d'autant plus complexe avec des apprenants dont le niveau de scolarisation et de littératie est souvent peu élevé, voire inexistant. Le corpus comprend donc des documents :

- écrits : documents administratifs, factures, bulletins scolaires, plannings, plans, tickets de caisse, abonnements, formulaires à remplir, prospectus publicitaires, quittances de loyer, notices de médicaments, annonces d'emploi, avis de passage, contrats de location, contrats de travail, annonces immobilières, etc. ;

- oraux : des enregistrements de conversations, de prises de rendez-vous, de demandes de renseignements, d'indications, d'itinéraires, de relations de services, d'échanges commerciaux, de bandes annonces (SNCF, par exemple), de boîtes vocales, de répondeurs téléphoniques, etc. ;

- vidéos : des séquences filmées d'interactions de services, d'échanges commerciaux, de rendez-vous administratifs, d'échanges quotidiens, etc. ;

- visuels : des photos d'affiches, de panneaux signalétiques, de panneaux indiquant des horaires d'ouverture et de fermeture, de plans, de lieux publics (mairie, boulangerie, école, préfecture par exemple), d'écrans de distributeurs d'argent, de plaques de médecins, de boittes aux lettres, de consignes de sécurité avec des pictogrammes, etc.

Enfin, les documents sont également classés selon les aptitudes ou les compétences langagières visées : Compréhension Écrite (CE), Expression Écrite (EE), Compréhension Orale (CO), Expression Orale (EO). Un même document peut permettre de travailler plusieurs aptitudes, mais nous avons fait le choix de catégoriser les documents par aptitudes séparées pour éviter, par exemple, le travail de l'écrit à partir d'un document oral. Nous avons encore rencontré récemment, lors de recherches sur le terrain en séances de formation, l'exemple classique de l'utilisation par un formateur d'un questionnaire à l'écrit pour évaluer la compréhension d'un document sonore.

${ }^{16}$ Nous pouvons rappeler que le niveau B1 à l'oral est exigé pour accéder à la nationalité française et que le niveau A.1.1. est exigé pour obtenir le DILF mais que l'examen comporte davantage d'épreuves de compréhension et d'expression orales. 


\subsection{Recueil et traitement des données}

Les données ont toutes été recueillies en situation naturelle ${ }^{17}$ et aucun document n'a été créé pour répondre aux besoins de l'échantillonnage du corpus ou encore aux besoins de données concernant tel ou tel point grammatical, par exemple. Les documents ont été sélectionnés en fonction de leur pertinence par rapport aux objectifs, aux enjeux et aux thèmes de l'apprentissage du français à visée d'intégration, comme nous l'avons expliqué précédemment. Les données ont également été recueillies indépendamment de leurs difficultés intrinsèques en termes, par exemple, de syntaxe, de lexique, de présentation des informations ou encore de registres de langue. Concrètement, pour les écrits et les visuels, nous avons recueilli les données susceptibles d'être rencontrées et/ou utilisées par les apprenants et, pour les audio et vidéo, susceptibles d'être proches des interactions vécues dans des situations de communication similaires.

Les exemples suivants ont été déposés dans FLIREP. Ils illustrent la variété des ressources disponibles et donnent un aperçu des différents traitements dont ils peuvent faire l'objet de la part des formateurs, en fonction des apprenants.

\section{Exemple 1:}

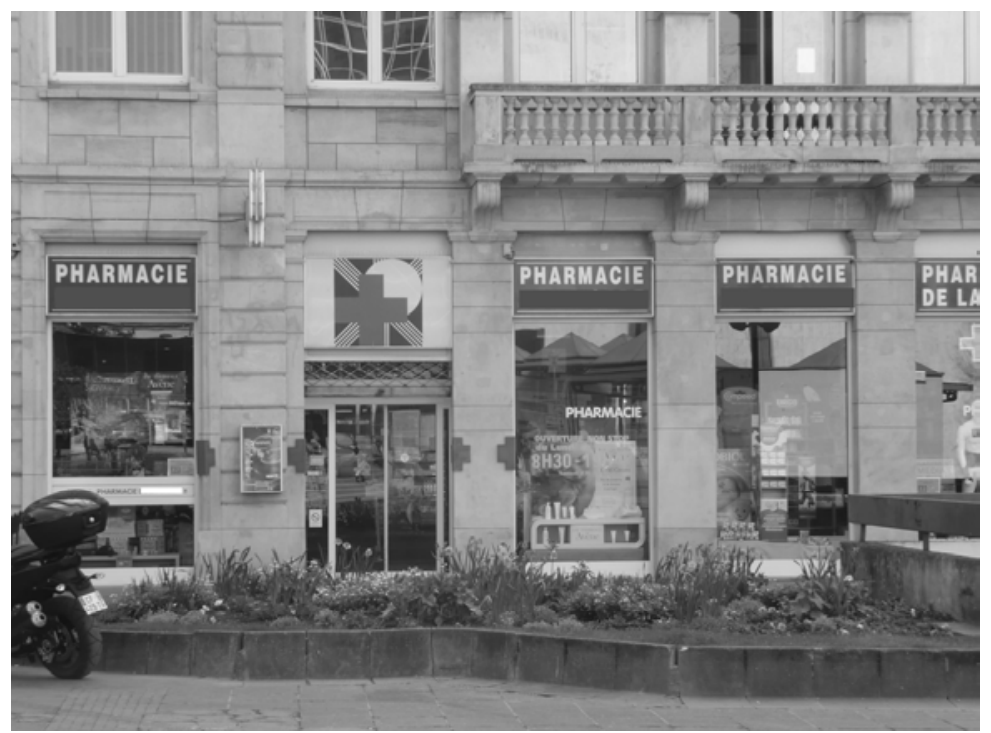

17 Tous les documents déposés dans la base de données font l'objet d'une validation par un coordinateur pédagogique. Au cours de notre expérimentation, nous avons confié différentes tâches de contrôle, vérification, expertise à des coordinateurs pédagogiques. Ces derniers sont chargés de coordonner les dispositifs de formation, de suivre les évolutions de la recherche en didactique et de veiller à l'adéquation des formations. 


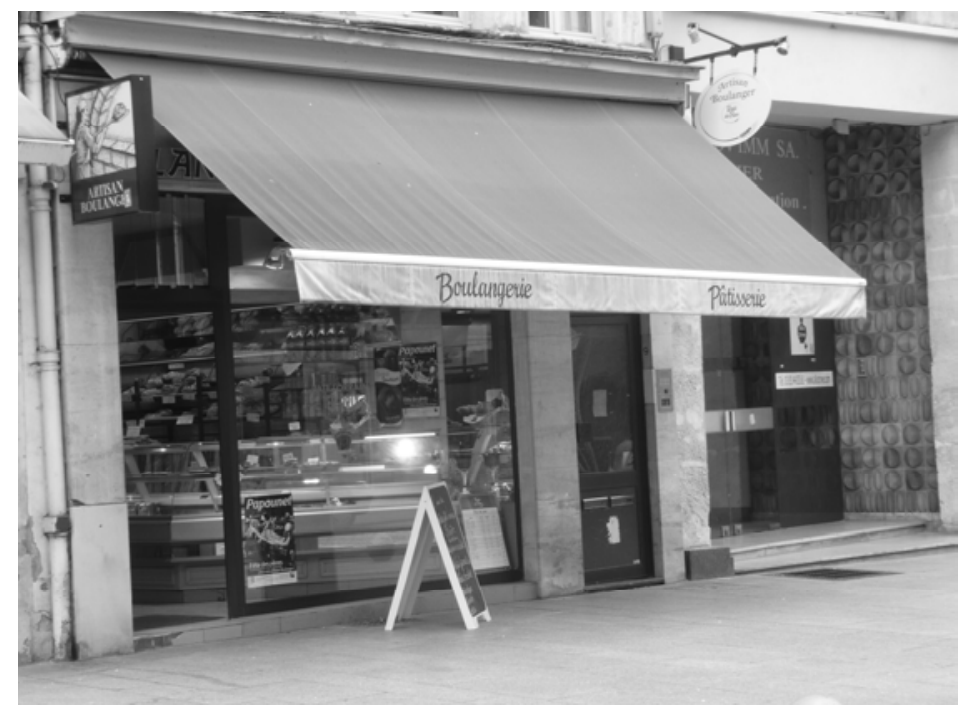

Ces deux photographies permettent aux formateurs de travailler la reconnaissance et l'identification des lieux susceptibles d'être fréquentés par les apprenants. Le corpus FLIREP comprend de nombreux visuels permettant de travailler avec les moins scolarisés et donc ceux qui sont les moins à l'aise avec l'écrit et les «technologies de l'intellect » (Goody, 2007). D'autres photographies, comportant légèrement plus de texte, ont été également recueillies : c'est notamment le cas des panneaux d'informations ou de la signalétique urbaine.

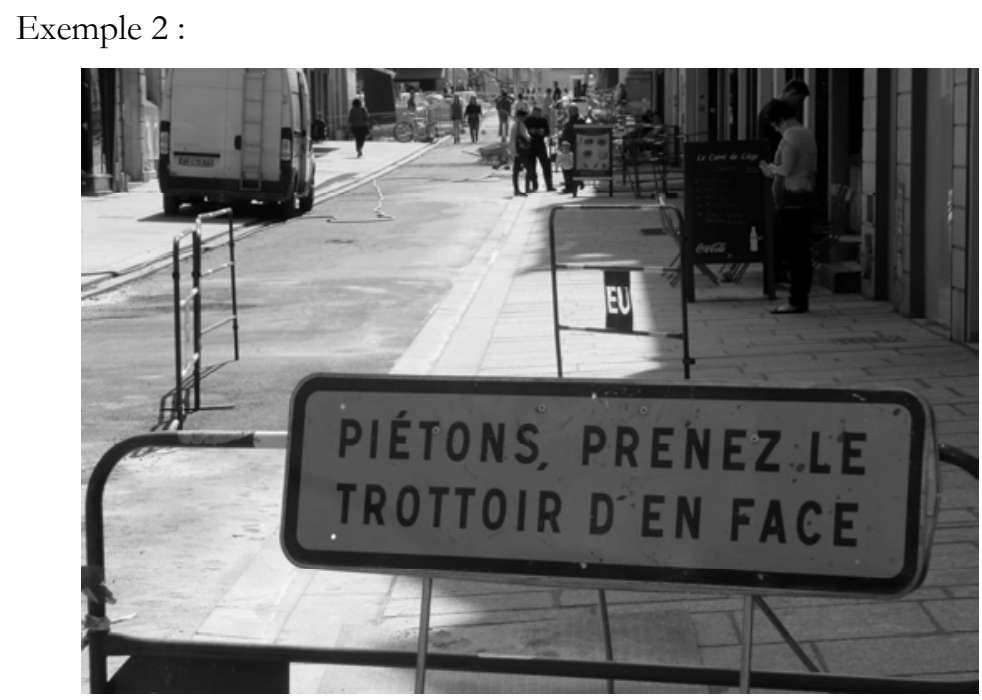


Les photographies, comme l'ensemble des ressources du corpus, sont toujours, de la meilleure façon possible, contextualisées, c'est-à-dire présentées avec un maximum d'éléments composant l'environnement dans lequel se trouve ces ressources. Ainsi, la photographie de l'exemple 2 fait non seulement apparaitre l'énoncé mentionné sur le panneau signalétique mais elle contextualise le panneau lui-même, la barrière sur laquelle il est fixé, la rue dans laquelle il se trouve. Nous tentons ainsi de reproduire les conditions dans lesquelles les apprenants sont susceptibles de rencontrer les ressources du corpus.

Les documents de compréhension écrite peuvent également être des petits mots laissés par des enseignants dans un cahier de liaison ou encore par des responsables sur un lieu de travail. L'exemple suivant est un texte affiché sur la porte d'une école élémentaire et destiné aux parents des élèves.

Exemple 3:

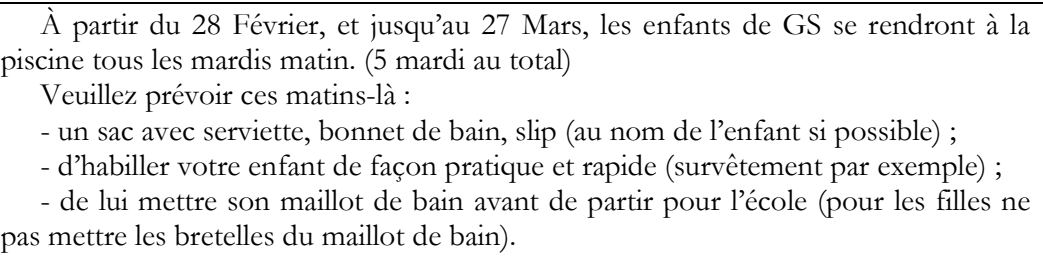

Ainsi, les documents écrits recueillis en situation peuvent être des photographies, permettant aux apprenants de reconnaittre un mot ou une expression, des textes courts, des textes plus longs ou des écrits spécifiques comme des factures, des contrats de travail ou encore des informations concernant la vie quotidienne.

Si les documents écrits sont souvent plus faciles à recueillir, les documents sonores et vidéo présentent des difficultés supplémentaires, en termes techniques et juridiques ou encore en termes de spontanéité (en fonction du mode de captation des données). L'exemple 4 est la transcription d'un document sonore déposé sur la plateforme avec les métadonnées suivantes : audio, consommation et service, après-vente, compréhension orale. Cet échange téléphonique a été enregistré en situation écologique par une formatrice qui cherchait réellement à faire réparer sa machine à laver par des professionnels.

Exemple $4^{18}$ :

L1 service après-vente *nom du magasin* bonjour

L2 oui bonjour je viens vous appeler pour avoir le tarif euh de la main d'œuvre pour une réparation hors garantie et je voulais savoir en fait euh parce que

\footnotetext{
${ }^{18}$ Nous avons choisi de transcrire les interactions orales orthographiquement, en respectant l'orthographe standard usuelle et en ne présentant aucun trucage. Cette transcription «nue » peut être enrichie par les formateurs qui le souhaitent afin, par exemple, de mettre en évidence des faits de prononciation ou des constructions syntaxiques particulières.
} 
c'est pour une machine à laver donc si euh je fais venir quelqu'un et que la machine coûte trop cher à réparer ou n'est pas réparable est-ce que si j'achète chez vous le devis est gratuit ou pas

L1 bon alors en fait quand on fait venir un technicien ça coute déjà vingt-quatre euros de déplacement

L2 oui

L1 donc les vingt-quatre euros quoi qu'il se passe que vous faisiez que vous faites réparer ou pas on vous remboursera pas les vingt-quatre euros par contre on vous remboursera les dix-huit euros qui correspondent à une demi-heure de main d'œuvre

L2 d'accord

L1 ça on vous rembourse si vous achetez effectivement une autre machine

L2 d'accord et euh

L1 mais pas les vingt-quatre

L2 ok

L1 voilà

L2 ben du coup est-ce que je peux prendre rendez-vous

L1 bien sûr il me faudrait la référence la marque de l'appareil

L2 oula alors attendez ben je pense que je vais vous rappeler parce qu'il faut que je le retrouve

L1 d'accord ou à moins que vous ayez la facture éventuellement

L2 elle a six mais il faut que je la retrouve parce que j’ai dû la ranger quelque part donc je vous rappelle d'ici cinq minutes quand j'ai retrouvé la facture

L1 pas de soucis

L2 à tout de suite

Ce type d'enregistrement, nous le répétons, peu fréquent dans d'autres corpus de données orales, représente une ressource utile et précieuse pour les formations FLI. Au-delà d'un travail possible sur le lexique, ce type de support audio permet de travailler la gestion de l'interaction, l'ouverture et la clôture de l'échange, la réalisation d'actes de parole spécifiques ou encore la construction syntaxique des questions à l'oral par exemple.

Comme nous l'avons précisé plus haut, peu de corpus déjà constitués contiennent des enregistrements d'interactions tronquées ou d'alteractions (Adami, 2012b). L'exemple suivant est la transcription du répondeur téléphonique d'un médecin (l'enregistrement est disponible sur le site) :

\section{Exemple 5 :}

bonjour vous êtes bien au cabinet du docteur Masson médecin généraliste le cabinet est ouvert du lundi au vendredi de huit heures à douze heures et de quatorze heures à dix-sept heures en cas d'absence vous pouvez essayer de me joindre au 06050403 
02 en cas d'urgence composez le 15 attention ce répondeur n'enregistre pas de message merci de votre appel

Pour effectuer des démarches administratives, les interactions se font de plus en plus avec des répondeurs téléphoniques qui orientent les appels vers un opérateur en fonction d'une série de questions-réponses automatisées. L'exemple suivant est un extrait de la boite vocale de la Caisse d'Allocation Familiale (CAF) :

\section{Exemple 6 :}

bonjour après le bip sonore cette communication sera facturée 16,8 centimes l'appel puis 2,8 centimes la minutes plus un coup depuis un mobile le détail est disponible au 3008 appuyez sur dièse pour ne pas écouter ce message (bip et jingle) bonjour bienvenue à la caisse d'allocations familiales de *département* (jingle) votre demande concerne l'allocation logement pour étudiant un numéro d'accès direct vous est désormais proposé le 0810292929 vous êtes allocataire tapez 1 si vous n'êtes pas allocataire de notre CAF tapez 2 si vous êtes partenaire bailleur tapez 3

Les documents, quelle que soit leur nature, sont anonymisés dans la limite de la pertinence de l'anonymisation. Ainsi, dans l'exemple 5, le nom du médecin a été changé mais dans l'exemple 6 , la «CAF» n'a pas été anonymisée, seul le département est supprimé de l'extrait sonore et de la transcription. Une réflexion, concernant les aspects juridiques des documents mis en ligne sur le site FLIREP, est en cours. Nous souhaiterions conserver, et ne pas anonymiser ou modifier, les logos notamment de la Poste, de la SNCF, de la RAPT, de la CAF, d'ERDF, de GRDF ou encore des banques dans la mesure où il est utile que les migrants les repèrent facilement.

\section{Exploitation du corpus}

\section{1. " Entrer " dans le corpus}

La recherche de documents dans le corpus est simplifiée au maximum pour les utilisateurs qui ne sont pas tous à l'aise avec la notion de corpus. Les métadonnées interrogeables pour « entrer» dans le corpus sont celles qui ont servi à l'échantillonnage du corpus. La recherche de documents s'effectue selon trois critères, pas nécessairement croisés :

- par type de document (écrit, audio, vidéo, visuel)

- par thème puis par sous thème

- par compétence langagière visée (CE, EE, CO, EO)

Une fois que le ou les documents sont choisis, ils peuvent être téléchargés, imprimés ou stockés dans un espace de travail sur le site. Les documents peuvent être bruts ou accompagnés d'une ou plusieurs exploitations pédagogiques, c'est-à-dire d'une ou plusieurs activités.

Les exploitations pédagogiques sont toujours associées à des ressources. Elles sont catégorisées selon les niveaux (du CECRL) et selon la compétence visée (CE, EE, $\mathrm{CO}, \mathrm{EO})$. Toutes les exploitations pédagogiques mutualisées sont créées par des 
formateurs et font l'objet d'une vérification par un coordinateur pédagogique avant la mise en ligne (elles sont généralement également testées par des formateurs). Les allers-retours entre le coordinateur et les formateurs participent de la formation de ces derniers.

Il existe donc plusieurs façons d'entrer dans le corpus, en choisissant l'ordre d'interrogation des métadonnées (thème, type de document, compétence langagière). Le Tableau 2 présente des exemples de recherches par critères. Les résultats des recherches multicritères sont présentés sous la forme d'une liste des ressources, classées par ordre alphabétique (selon la première lettre du titre du document).

Il est également possible d'accéder à la liste complète des ressources disponibles sur le site, classées par type de document (écrit, audio, vidéo, visuel). En outre, le titre des ressources est généralement assez significatif pour effectuer une recherche par titre ou par mot(s) du titre pour trouver un document correspondant aux attentes du formateur. Ces deux modes de recherche de documents sont encore possibles étant donné la taille du corpus. Avec l'augmentation du nombre de ressources, l'interrogation par métadonnées sera indispensable.

\begin{tabular}{|l|l|l|l|l|}
\hline & Critère 1 & Critère 2 & Critère 3 & Critère 4 \\
\hline Recherche 1 & $\begin{array}{l}\text { Thème } \\
\text { Logement }\end{array}$ & $\begin{array}{l}\text { Sous-thème } \\
\text { Gérer son logement }\end{array}$ & $\begin{array}{l}\text { Compétence } \\
\text { CE }\end{array}$ & \\
\hline Recherche 2 & $\begin{array}{l}\text { Compétence } \\
\text { CO }\end{array}$ & $\begin{array}{l}\text { Thème } \\
\text { Santé }\end{array}$ & $\begin{array}{l}\text { Sous-thème } \\
\text { Sécurité sociale et } \\
\text { mutuelle }\end{array}$ & \\
\hline Recherche 3 & $\begin{array}{l}\text { Thème } \\
\text { Consommation et } \\
\text { services }\end{array}$ & $\begin{array}{l}\text { Sous-thème } \\
\text { Impôts }\end{array}$ & $\begin{array}{l}\text { Type de } \\
\text { document } \\
\text { Andio }\end{array}$ & $\begin{array}{l}\text { Compétence } \\
\text { CO }\end{array}$ \\
\hline Recherche 4 & $\begin{array}{l}\text { Type de } \\
\text { document } \\
\text { Vidéo }\end{array}$ & $\begin{array}{l}\text { Thème } \\
\text { Citoyenneté et vie } \\
\text { en France }\end{array}$ & $\begin{array}{l}\text { Sous thème } \\
\text { Administration }\end{array}$ & \\
\hline
\end{tabular}

Tableau 2 : Exemples de recherches multicritères dans FLIREP

Le mode d'interrogation du corpus est, pour l'instant, assez sommaire. Les critères interrogeables méritent d'être précisés, affinés et augmentés au fur et à mesure de la saisie des documents du corpus dans la base de données et de leur analyse. L'expérimentation de FLIREP a déjà montré les limites de ce mode d'interrogation et a mis au jour l'importance de pouvoir croiser davantage de critères. L'analyse linguistique des données du corpus permet d'envisager, par exemple, un marquage pragmatique avec éventuellement une interrogation en termes d'actes de parole ou un marquage de phénomènes linguistiques spécifiques, avec une interrogation en termes de construction syntaxique, par exemple. 


\subsection{Une organisation repensée de la formation}

La mise en place d'une telle plateforme de mutualisation doit permettre, nous l'avons déjà dit, d'harmoniser les formations, d'offrir un outil souple et adapté au public hétérogène des migrants et de créer une véritable communauté de pratiques entre les formateurs (Wenger, 1998). Le site favorise en effet les échanges de pratiques (en observant, en analysant celles de ses collègues) et développe des relations entre les formateurs, même dans un contexte où les organismes de formation sont mis en concurrence par les multiples appels d'offre, privés comme publics. En outre, les pratiques des formateurs deviennent plus transparentes par la mise à disposition des documents et de leurs exploitations pédagogiques. Les actions de mutualisation permettent de faire converger les formateurs vers une démarche réflexive sur leurs expériences. Les trois dimensions propres à la communauté de pratiques, telle qu'elle est définie par Wenger (1998), sont l'engagement mutuel, l'action conjointe et le partage de connaissances. Ces aspects sont tout à fait présents et mis en œuvre dans le cadre de ce projet. Les formateurs s'engagent mutuellement et conjointement dans le partage de pratiques et de connaissances.

Ainsi, la nécessité d'utiliser des documents authentiques appropriés aux objectifs de la formation est non seulement facilitée par le site mais elle est également davantage acceptée par les formateurs grâce aux documents et aux exploitations pédagogiques de formateurs-experts servant d'exemples. Au fur et à mesure du recueil de nouvelles ressources, de la création de nouvelles activités, de l'observation des pratiques des autres formateurs et des aller-retour avec un coordinateur pédagogique, la démarche est harmonisée et gagne en qualité.

L'exploitation de ce corpus permet d'organiser différemment les séances de formation et d'appréhender l'hétérogénéité des groupes d'apprenants plus facilement. $\mathrm{Si}$, dans une situation de classe traditionnelle, il est extrêmement difficile de gérer l'hétérogénéité, la démarche didactique associée à l'utilisation du corpus permet de faire face à l'hétérogénéité des groupes d'apprenants. Cependant, dans la mesure où les formateurs peuvent choisir les documents selon des critères variés, ils peuvent tout à fait sélectionner un document différent pour chacun des apprenants présents en séance de formation. Les combinaisons sont multiples, par exemple :

- chaque apprenant travaille avec son propre document et ses propres activités pédagogiques ;

- des binômes ou trinômes, composés d'apprenants ayant des acquis différents mais partageant les mêmes objectifs, travaillent à partir du même document ;

- l'ensemble des apprenants travaille sur un même document avec des activités pédagogiques différentes ;

- etc.

Dans ces conditions, l'adaptation aux besoins des individus est possible (certains possèdent des acquis à l'oral, d'autres à l'écrit, certains possèdent des acquis à l'oral dans certains genres de discours, moins d'acquis dans d'autres genres, etc.). La 
modularité et l'individualisation deviennent concrètement réalisables. Une même séance de formation peut même faire alterner des phases d'individualisation et des phases de travail collectif.

Cette démarche, avec des phases d'individualisation, de travail en petits groupes ou de travail collectif, permet de réduire également un phénomène fréquemment rencontré lors de formations linguistiques pour des adultes migrants, même si ce n'est pas spécifique à ce champ didactique : la passivité de certains apprenants. Adapter la formation aux apprenants permet de les rendre actifs et évite qu'ils ne subissent la formation, notamment, par exemple, en comptant sur le voisin qui est légèrement plus avancé et qui finira donc bien par réussir l'activité.

\subsection{Exploitation pédagogique du corpus}

Les ressources du corpus sont accompagnées d'une ou plusieurs exploitations pédagogiques créées par des formateurs et validées par un coordinateur pédagogique avant leur mise en ligne. Ces exploitations ou activités pédagogiques sont conçues en fonction des caractéristiques des apprenants. Par exemple, en fonction de leur niveau de scolarisation, les apprenants ne pourront pas réaliser le même type d'activité et les documents devront être abordés de manière spécifique.

Chaque exploitation pédagogique précise le niveau du CECRL visé : A1.1, A1, A2 ou B1. À une même ressource (écrite, audio, vidéo et visuelle) peuvent être associées plusieurs exploitations pédagogiques de même niveau ou de différents niveaux. Un même formateur peut créer plusieurs activités de même niveau ou des activités de différents niveaux parce qu'il aura souhaité travailler un même document avec des apprenants différents ou plusieurs formateurs créent chacun une ou plusieurs activités de niveaux différents et la mutualisation augmente le choix des exploitations pédagogiques.

Nous pouvons mentionner également au moins deux façons de procéder pour exploiter ce corpus :

- le formateur réalise une analyse des situations de communication rencontrées par les migrants en termes d'éléments situationnels, d'actes de paroles ou de compétences langagières, d'aptitudes (CE, CO, EE, EO) et de pratiques langagières. Sur la base de cette analyse, le formateur peut rechercher le ou les documents appropriés dans la base de données ;

- le formateur peut analyser les documents déjà recueillis, en termes de situations de communication, d'actes de paroles ou de compétences langagières et de pratiques langagières. Il estime le degré de compatibilité entre les documents analysés et les besoins et les objectifs de ses apprenants.

L'outil FLIREP se caractérise par sa grande souplesse et les formateurs peuvent entrer dans le corpus de différentes façons et peuvent l'exploiter en fonction de leurs pratiques, de leurs apprenants, de leurs conditions matérielles de travail, etc. 


\section{Conclusion}

Le champ didactique de la formation linguistique des migrants s'inscrit dans un processus de structuration pédagogique, institutionnel et professionnel qui ne cesse de se renforcer depuis les années quatre-vingt-dix (Leclercq, 2012). Le dispositif FLI arrive au bout de ce processus et a pour objectif principal l'amélioration de la qualité des formations linguistiques pour les adultes migrants. Les problèmes particuliers que pose la didactique du français pour les adultes migrants n'ont que très rarement été traités par la didactique du FLE et du FLS. Nous avons déjà souligné (Adami, 2012a) l'indigence des recherches dans ce domaine et le peu d'intérêt que la didactique du FLE/FLS a accordé à la formation linguistique des migrants adultes, préférant se consacrer aux domaines mieux connus de l'école et de l'université. La polémique sur le FLI a mis pour un temps ce champ didactique sur le devant de la scène mais une fois l'agitation retombée et les opinions exprimées, nous constatons toujours aussi peu d'affluence scientifique sur le terrain. Ce terrain, c'est plus de cinq-cents organismes de formation professionnalisés en France, des centaines d'associations de quartier et des milliers de formateurs. Dans ce cadre, nous essayons d'apporter notre modeste contribution théorique et pratique avec cette recherche-action. C'est une innovation fondamentale parce qu'elle doit permettre de contribuer à résoudre le problème central en formation linguistique des adultes migrants, à savoir l'hétérogénéité des groupes. Elle pourrait donner lieu, après que nous en aurons mesuré la portée et l'efficacité didactiques, à d'autres expérimentations sur le terrain. Seule une étude à moyen voire à long terme pourra nous renseigner sur l'adéquation et l'efficacité de la démarche. En outre, au-delà des préconisations liées à l'exploitation de ce corpus, se posent d'autres questions, liées aux pratiques réelles des formateurs ainsi qu’à leur formation. Mais ceci est encore un autre chantier...

Notre travail de recherche-action fait état ici d'une initiative particulière mais il s'inscrit de toute façon dans la durée. C'est notre présence constante sur le terrain et avec les acteurs qui permet de mettre nos différentes recherches en perspective, sans les dissocier les unes des autres mais au contraire en les articulant. Nous aurons donc à mesurer les effets à court, moyen et long terme de l'introduction de ce corpus en formation linguistique des migrants adultes. 


\section{Bibliographie}

ADAmi, H. (2012a), « La formation linguistique des migrants adultes », Savoirs, 29/2, p. 9-44.

ADAMI, H. (2012), «Aspects sociolangagiers de l'acquisition d'une langue étrangère en milieu social », dans H. Adami \& V. Leclercq (éds.), Les migrants face aux langues des pays d'accueil. Acquisition en milieu naturel et formation, Villeneuve d'Ascq, Presses Universitaires du Septentrion, p. 51-85.

ADAMI, H. (2010), «L'acculturation linguistique des migrants: des voies de l'insertion sociolangagière à la formation", dans L. Cadet, J. Goes \& J.-M. Mangiante (éds.), L'intégration et la formation linguistique des migrants : état des lieux et perspectives, Arras, Artois Presses Université, p. 37-54.

ADAMI, H. (2009), «Les documents authentiques dans la formation des adultes migrants: pratiques pédagogiques et contraintes institutionnelles », Mélanges CRAPEL, 31, p. 159172.

ADAmI, H. (2005), «Français Langue Etrangère et enseignement du français aux migrants : les faux jumeaux didactiques", Le français dans le monde, 339, p. 23-26, http://www.fdlm.org/fle/article/339/adami.php.

AdAmi, H., \& ANDré, V. (2012), «Vers le Français Langue d'Intégration et d'Insertion (FL2I) », dans H. Adami \& V. Leclercq (éds.), Les migrants face aux langues des pays d'accueil. Acquisition en milieu naturel et formation, Villeneuve d'Ascq, Presses Universitaires du Septentrion, p. 277-289.

ANDRÉ, V. \& CANUT, E. (2010), «Mise à disposition de corpus oraux interactifs : le projet TCOF (Traitement de Corpus Oraux en Français) », Pratiques, 147-148, p. 35-51.

CUQ, J.-P. \& GRUCA, I. (2002), Cours de didactique du français langue étrangère et seconde, Grenoble, Presses Universitaires de Grenoble.

CANDIDE, C. (2001), «Apprentissage de la langue : vers une lente émergence d'un droit », VEI enjeux, 125, p. 108-117.

De Pietro, J.-F., MAtThey, M. \& CONTI, V. (2012), « Langue, intégration, cohésion sociale... Quel rôle pour une politique linguistique », dans V. Conti, J.-F. De Pietro \& M. Matthey (éds.), Langue et cohésion sociale. Enjeux politiques et réponses de terrain, Neuchâtel, Délégation à la langue française, p. 9-33.

EXtramianA, C., (2012), «Les politiques linguistiques concernant les adultes migrants », dans H. Adami \& V. Leclercq (éds.), Les migrants face aux langues des pays d'accueil. Acquisition en milieu naturel et formation, Villeneuve d'Ascq, Presses Universitaires du Septentrion, p. 135-152.

Glohguen-Vernet, N. (2009), Enseigner le français aux migrants, Paris, Hachette Français Langue Etrangère.

GoOdy, J. (2007), Pouvoirs et savoirs de l'écrit, Paris, La Dispute.

Holec, H., (1990), «Des documents authentiques, pour quoi faire?», Mélanges pédagogiques, 1990, p. 65-74. 
Hervé Adami \& Virginie André

LANGBACH, V. (2014), Analyse et mesure des insécurités langagières chę des adultes en situation d'insertion, Thèse de doctorat sous la direction de Hervé Adami et Virginie André, Université de Lorraine.

LECLERCQ, V. (2012), «La formation des migrants en France », dans H. Adami \& V. Leclercq (éds.), Les migrants face aux langues des pays d'accueil. Acquisition en milieu naturel et formation, Villeneuve d'Ascq, Presses Universitaires du Septentrion, p. 173-196.

LECLERCQ, V. (2007), « La formation de base : publics, dispositifs, pratiques », Savoirs, 14, p. 855.

Mourlhon-Dallies, F. (2008), Enseigner une langue à des fins professionnelles, Paris, Didier.

NoIRIEL, G. (1988), Le creuset français. Histoire de l'immigration XIXe-XXe siècle, Paris, Seuil.

SCHNAPPER, D. (2007), Qu'est-ce que l'intégration ?, Paris, Gallimard.

Veillette, J. (2013), «L'immigration dans de petites villes 'bilingues' fribourgeoises, ou quand l'acquisition du français est révélatrice de logiques locales particulières », dans C. Hauser, P. Milani, M. Pâquet \& D. Skenderovic (éds.), Sociétés de migrations en débat. Québec-CanadaSuisse : approches comparées, Laval, Presses de l'université Laval.

Wenger, E. (1998), Communities of Practice. Learning, Meaning and Identity, Cambridge, Cambridge University Press.

Wihtol De Wenden, C. (2008), « Migration, pluralité, intégration », Glottopol, 11, p.13-22. 